In press: International Perspectives in Psychology: Research, Practice, Consultation. (C) 2017, American Psychological Association. This paper is not the copy of record and may not exactly replicate the final, authoritative version of the article. Please do not copy or cite without authors permission. The final article will be available, upon publication, via its DOI: 10.1037/ipp0000074

\title{
Cooperation and Trust in Japanese and British Samples: Evidence from Incomplete Information Games
}

\section{Eva M. Krockow, Masanori Takezawa, Briony D. Pulford, Andrew M. Colman, and Toshimasa Kita}

\author{
Author Note \\ Eva M. Krockow, Briony D. Pulford, and Andrew M. Colman, Department of
} Neuroscience, Psychology and Behaviour, University of Leicester; Masanori Takezawa, Centre for Experimental Research in Social Sciences and Department of Behavioral Science, Hokkaido University; Toshimasa Kita, Department of Behavioral Science, Hokkaido University

We are grateful to the Leicester Judgment and Decision Making Endowment Fund (Grant RM43G0176) and Friedrich-Naumann-Foundation for Freedom for support in the preparation of this article and to Jodil Davis for developing the software used in the experiments.

The anonymized, raw data set of the experiment is available from the authors upon request. Correspondence concerning this article should be addressed to Eva M. Krockow, Department of Neuroscience, Psychology and Behaviour, University of Leicester, Leicester LE1 7RH, United Kingdom. E-mail: emk12@le.ac.uk

\begin{abstract}
Most human relationships are characterized by reciprocal patterns of give-and-take that can be studied using a decision making task called the Centipede game. The game involves two players alternating in choosing between cooperation and defection, with their choices affecting payoffs to themselves and the co-player. We compared trust and cooperation of Japanese and UK samples in the Centipede game. To increase the game's applicability to real-life decision situations, we added three treatment conditions to manipulate payoff information. Our betweensubjects design comprised the following four conditions: (a) full payoff information, (b) full payoff information framed as percentages, (c) partial payoff information with absolute (own payoff) information only, and (d) partial payoff information with relative information only. Comparing Japanese and UK students' decisions, the Japanese cooperated significantly more frequently than the British. The manipulation of payoff information also affected decision making. In Japan, both treatment conditions with incomplete information yielded significantly higher cooperation levels than the control. In the UK, only the condition with absolute payoff information produced significantly higher cooperativeness. Overall, these findings suggest that Japanese samples cooperate more frequently in repeated interactions than British samples and that this may be due to the assurance-based trust elicited by reciprocal relationships that has been identified as a typical feature of Japanese culture. In situations with incomplete information, expectations about the stake size may guide decision making, with lower expectations resulting in higher cooperation levels.
\end{abstract}

Keywords: Centipede game, cooperation, reciprocity, incomplete information, social value orientation, trust 


\section{Cooperation and Trust in Japanese and British Samples: Evidence from Incomplete Information Games}

In the face of rapid globalization, international collaboration is key for ensuring economic growth and progress. Consider, for example, two international business firms that take turns in sharing expertise in order to design a new product. Cooperation in the form of investing personnel and resources is always costly in the short term and potentially risky if the other firm does not reciprocate. However, mutual and repeated cooperation could lead both firms to launch the new product successfully and flourish in the long run. Business collaborations of this type are frequent and often take place across continents. Examples are partnerships between the US computer and Japanese automotive industry as well as UK-Japanese collaborations in the robotics sector.

In these global examples of repeated, reciprocal interactions, cultural differences may play an important role that could affect cooperation and, ultimately, the success of international collaborations. Japanese society, for example, is historically characterized by close-knit communities with strong interpersonal bonds relying on assurance-based trust, examples of which include highly committed, loyal interactions within business groups (so-called keiretsu) and typically lifelong employment with the same company (e.g., Kim, Hoskisson, \& Wan, 2004; Spencer \& Qiu 2001). Western entrepreneurship, on the other hand, has been found to place a stronger emphasis on innovation, for example through staff turnover (Tiessen, 1994). These different understandings of business management alongside intercultural variations in prosociality, risk-taking, and trust may be important factors determining the success of international business relationships.

\section{Centipede Game}

To enable the study of cooperative turn-taking as described above, Rosenthal's (1981) Centipede game offers a tool for experiments in a controlled laboratory environment (see Figure 1). The game provides a dynamic model for reciprocal human relationships, where Player A and Player B-representing either individual decision makers or larger entities such as groups, business firms, or even states_-alternate in deciding whether to cooperate (e.g., invest expertise and resources in a joint project) or to defect (e.g., exit a business partnership). The interaction starts at the leftmost decision node of the branched diagram (game tree) and proceeds to the right in case of a cooperative move (GO) or down (defect) in case of a non-cooperative move (STOP). Player A can make decisions at odd numbered decision nodes and Player B at even numbers. If both players choose GO at every decision node, then the game reaches a natural end on the right. In the example game, the end is reached following a GO move at decision Node 8. Each STOP move terminates the game and leads to an exit node, where the game's final outcome is shown. Player A's payoff is displayed above Player B's in each case. For reasons of simplicity, these payoffs are expressed in monetary units that could represent or be converted into any currency (e.g., British Pounds Sterling or Japanese Yen). 


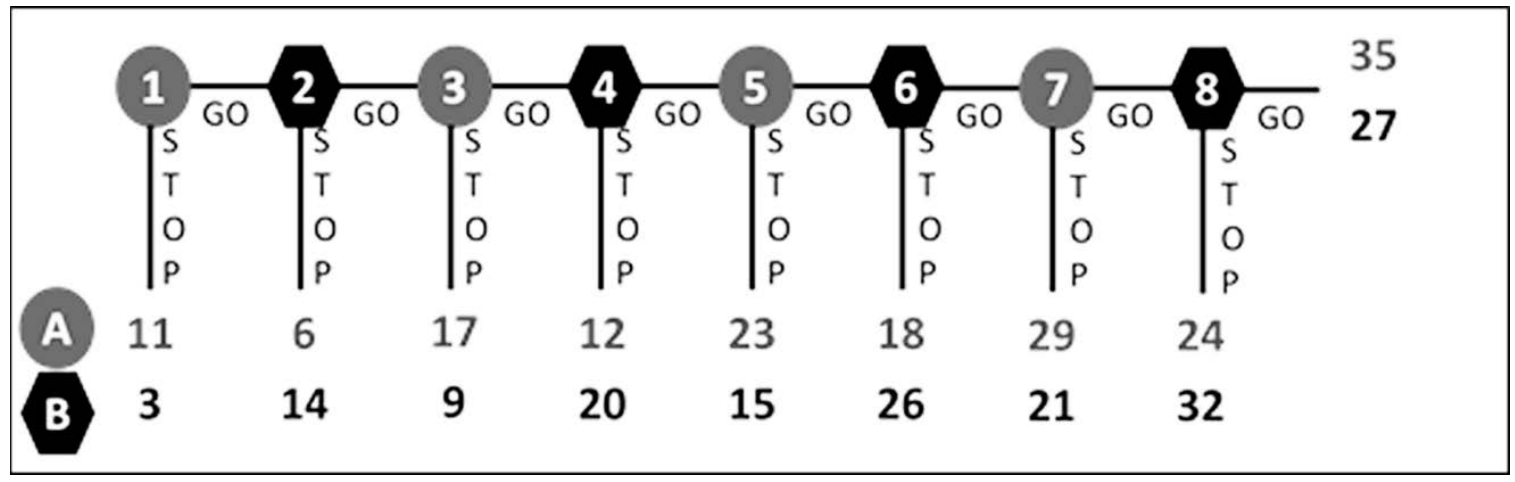

Figure 1. Example Centipede game.

Looking at the payoff function of the game, a cooperative GO move serves to maintain the relationship of the two players. It benefits the other player-each personal GO move adds 11 monetary units to the co-player's payoff-as well as the team, since the two players' joint payoff increase by 6 monetary units at each node that is passed. Nevertheless, a cooperative decision always incurs a cost of 5 monetary units to the individual who cooperates. Defection, on the other hand, always terminates the interaction with a favorable payoff to the defector-in the example game, the defector always earns 8 monetary units more than the co-player.

Thus, the Centipede game is uniquely suited to investigating dynamic, sequential decision problems with accumulating stakes across time-decision problems in which the applicability of better-known economic games such as the one-shot Prisoner's Dilemma game is limited. Whereas the standard Prisoner's Dilemma can be used to investigate cooperation in singular and discrete choice dilemmas, the Centipede game provides a model for potentially indefinite reciprocal relationships. Some of the Prisoner's Dilemma's shortcomings are overcome in the iterated or repeated Prisoner's Dilemma game, a dynamic variation comprising a series of identical one-shot Prisoner's Dilemmas with the same co-player. However, even in this dynamic game version, decisions are still made simultaneously without knowledge of the other player's choice. This property renders the game an unsuitable model of the type of sequential reciprocal cooperation being considered here. The Centipede game is also characterized by a theoretically interesting antagonism between the incentive for long-term mutual cooperation, potentially resulting in increasingly high payoffs to both players, and the short-term temptation to defect, yielding immediate payoffs with a generous share to the defecting player. Reaching the game's natural end relies on a purely altruistic move by Player B, because the final GO move sacrifices some of the cooperating player's personal payoff for the good of Player A and the team without the opportunity for Player A to return the favor. In fact, traditional game theory claims this final GO move to be irrational and constructs a mathematical argument around it that ultimately rules out any cooperation at all in the Centipede game, instead mandating an immediate STOP move at Node 1 (see Aumann, 1995, 1998; Colman, Krockow, Frosch, \& Pulford, 2017, for detailed discussions of the so-called backward induction argument).

A number of experimental studies have been conducted on the Centipede game, with most reporting high levels of cooperation and games frequently reaching their natural ends (e.g., McKelvey \& Palfrey, 1992; Pulford, Colman, Lawrence, \& Krockow, 2017). A variety of factors of the game design have been shown to influence decision making, including payoff function (e.g., Fey, McKelvey, \& Palfrey, 1994; Krockow, Pulford, \& Colman, 2015), stake size 
(Rapoport, Parco, Stein, \& Nicholas, 2003), and the number of players taking turns in the game (Murphy, Rapoport, \& Parco, 2006).

Despite the game's importance for modeling reciprocal relationships as outlined above, no cross-cultural research has been published on the Centipede game so far, and only one study has been conducted using an Asian sample (Kawagoe \& Takizawa, 2008). In the context of global business partnerships, of particular interest for comparison are industrialized nations with distinctly different religious and cultural backgrounds including, for example, the United Kingdom contrasted with Japan (Hofstede, 2001b).

\section{Cross-Cultural Differences Between Japan and the UK}

A concept that has received much theoretical and empirical attention in the context of economic growth and development is the cultural dimension of individualism versus collectivism (e.g., Hofstede, 2001a; Hofstede, Hofstede \& Minkov, 2010; Triandis, 1995). This refers to the respective focus on either the individual or the collective within society. Japan was found to score comparatively low on individualism (although not as low as neighboring Asian countries such as China or Vietnam), pointing to a relatively small cultural emphasis on personal independence (Hofstede, 2010). According to Hofstede's survey data, on the other hand, the UK was characterized by one of the highest individualism scores in the world (exceeded only by other Anglo countries such as the US and Australia), thus indicating strong values of individual autonomy. However, Hofstede's (2010) original research on the topic, conducted from the 1960s onwards using highly educated participants (all skilled employees of the international company IBM), has been challenged on a number of grounds, including the representativeness of national samples (e.g., Schwarz, 1994; Voronov \& Singer, 2002) and the use of self-report measures with individualist or collectivist cultural values that have been claimed to lack the necessary explicitness for self-report (e.g., Oyserman, Coon, \& Kemmelmeyer, 2002). Additionally, Voronov and Singer (2002) questioned the dimension's theoretical validity, and in particular its assumed bipolarity, with individualism and collectivism being presented as two mutually exclusive cultural values at opposite ends of a spectrum. For example, Ho (1993) argued that collectivism (in Asian countries) places a greater emphasis on personal relationships than the welfare of an entire social group, and therefore does not necessarily oppose individualism.

Additionally, country-specific research has demonstrated inconsistencies in Japanese individualism scores, with trends toward higher scores over time (Matsumoto, 1999; Takano \& Osaka, 1999). Indeed, Yamagishi (1988a, 1988b) questioned the concept's usefulness in the context of Japanese culture. Yamagishi employed public goods games, with opportunities for sanctioning, to explore collectivism in Japanese and American people, and found that behavioral differences in cooperation were not based on different values but could instead be explained by societal structures (e.g., the existence of strict rules and opportunities for punishment that deter defection in Japanese society). In an evaluative review, Voronov and Singer (2002, p. 474) concluded: "Clearly, individualism and collectivism do not exist within people's minds but, rather, manifest themselves in people's behavior, which is determined by the social context."

Consequently, it has been suggested that the Japanese type of rule-enforced collectivism is better conceptualized as assurance or assurance-based trust, serving the individual's personal gains in the long run (Voronov \& Singer, 2002; Yamagishi, 1988a, 1988b; Yamagishi \& Yamagishi, 1994). Indeed, for the comparison of economic decision making between Japanese and British nationals, the individualism/collectivism dimension may not be the most useful concept, and a focus on cultural differences in trust may be more fruitful. 
Yamagishi and Yamagishi (1994) differentiated two types of trust, each predominant in different cultures. They distinguished between general trust exhibited toward strangers, with higher prevalence in Western culture, and assurance-based trust effective in stable, committed relationships with higher prevalence in Eastern-particularly Japanese-culture. According to this distinction, assurance-based trust does not necessitate a belief in the benevolence of the other person but is based on the "knowledge of the incentive structure surrounding the relationship" (Yamagishi \& Yamagishi, p.132).

This theory of trust has been supported by a number of empirical studies. In Hayashi et al.'s (1999) cross-cultural experiment on Prisoner's Dilemma games, cooperation of Japanese participants was significantly improved by introducing a sense of control. Using sequential versions of the original game, where the two players decided one after the other whether to cooperate or to defect, they compared the choices of Player 1 and Player 2 in Japan and the US, either with or without perfect information about co-players' moves. In contrast with the American sample, Japanese participants in the role of Player 1 cooperated significantly more often under conditions of perfect information than in the other treatment conditions. This was attributed to a sense of control experienced by the first decision makers, who expected their own choices to influence co-players' subsequent actions, with a cooperative move anticipated to elicit reciprocity.

Similarly, Kiyonari, Yamagishi, Cook, and Cheshire (2006) conducted a US-Japanese comparison of decision making in Trust games. After two players received the same initial endowment, Player 1 had to decide whether to play safe by keeping the endowment or to trust the co-player by handing over the endowment, which would subsequently be doubled by the experimenter. Upon receipt of Player 1's doubled endowment, Player 2 then had the choice between either reciprocal cooperation, by returning a share of the doubled endowment to Player 1, or defection, in which case Player 2 kept the full amount. In the American sample, more trust was exhibited by Players 1 and less reciprocity was shown by Players 2 than in the Japanese sample, suggesting that general trust was higher but trustworthiness and reciprocity were lower than in Japan. Extending this study's findings, Kuwabara et al. (2007) found higher levels of general trust exhibited by US than Japanese players in a variation of the Trust game played with strangers. However, the Japanese sample showed increased trust when each participant was repeatedly paired with the same co-player.

Taken together, the above findings demonstrate that Japanese participants, in contrast with US participants, cooperate more frequently in decision situations (a) when they have an increased sense of control; (b) when their decision is preceded by a cooperative move of the coplayer; and (c) when they find themselves re-matched with a previous, and thus familiar, coplayer. This lends support to Yamagishi and Yamagishi's (1994) trust theory, according to which assurance-based trust - necessary for stable relationships - is more prevalent in Japan than in Western cultures. It also appears that Yamagishi and Yamagishi's understanding of assurancebased trust as an ecological or structural guarantee of cooperation permeates all Japanese relationships with a relevant interpersonal history of bilateral, reciprocal cooperation, even in the absence of formalized incentives for cooperation or deterrents of defection.

Finally, the proposed differentiation in trust shows interesting parallels with selfconstrual theory (Markus \& Kitayama, 1991; Voyer \& Franks, 2014). This theory proposes different types of self-concepts: People with more independent self-concepts place a stronger emphasis on their personal characteristics, while people with more interdependent self-concepts define themselves more frequently in terms of successful relationships. Self-construal theory has 
been widely used in applied social and cognitive psychology (e.g. Howard, Gardner, \& Thompson, 2007; Mandel, 2003). Furthermore, research has suggested that Asian cultures tend to generate more interdependent self-concepts (e.g., Christopher \& Skillman, 2009) that would foster assurance-based trust as claimed by Yamagishi and Yamagishi.

\section{Study Aims}

Although many studies (e.g., Berigan \& Irwin, 2011; Cox, Lobel, \& McLeod, 1991; Wong \& Hong, 2005) have investigated the relationship between culture and cooperation, as far as we are aware, no previous studies comparing British and Japanese decision making in dynamic relationships have been published. The present study and a related experiment currently under review for publication (Krockow, et al., 2017) follow up on the findings from USJapanese cross-cultural studies on short sequential games. One aim is to extend the research by using longer game interactions - Centipede games-allowing for investigation of longer-term reciprocal relationships. Centipede games may, in fact, be highly relevant to Japanese society, as they arguably provide much closer models of the tight family and business bonds on which Yamagishi and Yamagishi (1994) based the Japanese concept of trust. Indeed, when looking at the above factors (a) to (c) that were found to elicit Japanese assurance-based trust in previous experiments - sense of control, opportunity for reciprocity, and repeated interaction with the same other player - the Centipede game appears to be a decision context that combines them all. Arguably, an initial amount of general trust comes into play when first entering the Centipedetype interaction with a complete stranger. As mentioned above, previous research found hardly any participants who ended the interaction at the first decision node (McKelvey \& Palfrey, 1992), and qualitative research by Krockow, Pulford, and Colman (2016) suggested the early activity bias, curiosity, and a wish to probe the co-player were at least partly responsible for the low termination rates at Node 1. Although the relationships between players were not manipulated in this experiment, initial concerns of general trust seem to be overruled by different motives, and the more predictive type of trust for decision making in Centipede games is likely to be the assurance-based trust of stable partnerships.

In order to further increase the study's applicability to real-life decision contexts, we introduced treatment conditions with incomplete information in addition to the standard Centipede game. The bulk of research on experimental games has focused on decision-making contexts with clearly defined rules, known payoffs, and complete information of these. Complete information is achieved if all decision makers involved in the game are informed about all rules of the game and the payoffs to themselves and all other players in every possible outcome of the game, and if all players know that all players know that all players know these facts, and so on ad infinitum.

Nevertheless, games with limited payoff information may be much closer models of reallife decision situations, where certain aspects of an interaction's possible outcomes may be unknown or at least uncertain. Only very few studies, including Croson's (1996) study on Ultimatum games, have investigated experimental games with partial information. In an Ultimatum game, two participants are assigned to different roles_-Proposer and Responder-and the former receives an initial endowment that has to be shared with the Responder. Following the Proposer's decision regarding the proportion of the share, the Responder has to decide whether to accept or reject the offer, with rejection resulting in zero payoffs to both players. Croson found that if the Responders in the Ultimatum game did not know the overall pot of money at stake, then the offers made by the Proposers were significantly lower than offers in a control condition where the size of the pot was known. The study further showed that in Ultimatum games with 
complete payoff information, offers presented as percentages (rather than as absolute payoffs) led to a significant increase in rejection rates and overall higher demands by the Responders, possibly because the relative payoff information increased inter-player comparisons and resulted in stronger considerations of fairness. Taken together, these findings indicate that the amount of payoff information as well as the framing of this information, either as absolute payoffs (total values) or as relative payoffs (percentages), could affect decision making in experimental games.

Our study aimed to follow up on Croson's (1996) experiment and to test for the effects of incomplete payoff information in the Centipede game. In particular, we compared (a) standard Centipede games (Condition 1); (b) complete-information games where the payoffs were framed as percentages (Condition 2); (c) incomplete-information games where participants knew only their personal absolute payoffs (Condition 3); and (d) incomplete-information games where participants received only relative payoff information about their share of an unknown pot of money (Condition 4). Whereas we did not expect significant differences between Treatment Conditions 1 and 2, as both are characterized by complete payoff information, ${ }^{1}$ albeit framed in different ways, we formulated the following hypotheses regarding significant influences of the treatment conditions.

\section{Hypotheses}

We set out to conduct what appears to be the first experimental comparison of economic decision making between Japanese and British culture. Based on the literature reviewed above, we formulated the following hypotheses.

Hypothesis 1. Given the higher levels of assurance-based trust necessitated by long-term, reciprocal interactions modeled by the Centipede game, Japanese participants will exit later and thus exhibit higher cooperation levels in the Centipede game than UK participants. These later exit moves in Japan will also be reflected in a higher proportion of games reaching the final end.

Hypothesis 2. As a result of the decreased predictability of the co-players' moves in games with incomplete payoff information and the consequent riskiness of cooperative GO moves, Centipede games with incomplete payoff information will yield lower levels of cooperation than games with complete information.

Hypothesis 3. Treatment Condition 4, with relative payoff information only, will result in particularly low cooperation levels due to the increased emphasis on player inequality, which taps into prosocial concerns for fairness.

Additional hypotheses relating to individual differences are introduced below. Before stating these, we review relevant literature related to individual differences.

\section{Individual Differences}

In order to pinpoint reasons other than assurance-based trust for any behavioral differences between countries and treatment conditions in the study, we aimed to assess a variety of individual difference variables. Very few Centipede studies to date have investigated personality variables and their effects on decision making. However, two experiments by Pulford, Krockow, Colman, and Lawrence (2016) and a qualitative study on decision making in the Centipede game (Krockow, Pulford, \& Colman, 2016) identified Social Value Orientation (SVO) as a key variable in the context of the reciprocal interactions. SVO categorizes individuals depending on their preferences regarding the distribution of (financial) resources between themselves and another person (e.g., Balliet, Parks, \& Joireman, 2009; Messick \& McClintock, 1968). The most frequent type of SVO is the prosocial orientation, an umbrella term including cooperative individuals striving to maximize the team's earnings. Other prosocial SVO sub-types are the altruistic orientation, striving to maximize the other person's payoff, and the equality- 
seeking orientation, striving to achieve payoff equality between themselves and the other person. Other SVOs are the competitive orientation, striving to increase one's own share of resources relative to the other person's, and the individualistic orientation, striving to maximize one's own individual payoff irrespective of the other person's. In general, prosocial SVO was found to be related to more cooperative play in the Centipede game-particularly toward the end of the game-whereas individualistic SVO was more frequently related to STOP moves and thus lower cooperation levels in the game (Krockow, Pulford, \& Colman, 2016).

In Japan, only one comprehensive study to date has tested for the influence of SVO on decision making in experimental games (Yamagishi et al., 2013). The study identified very few participants with altruistic and competitive SVOs, and the number of cooperative individuals seemed slightly higher than those with individualistic categorizations, a result corroborating the general SVO findings for Western samples (Balliet, Parks, \& Joireman, 2009). The Japanese results, however, lacked conclusiveness due to the use of categorical SVO measures (Ring measure and Triple-Dominance measure) that were found to have poor test re-test reliability (the authors reported $57.7 \%$ for the Ring measure) and did not show a strong correlation across questionnaires $(r=.38)$. To overcome previous measurement problems, the study reported in this article used the more recently introduced continuous "Slider measure" of SVO, which provides a more accurate and reliable assessment of SVO than previous categorical measures (Murphy, Ackermann, \& Handgraaf, 2011).

Another individual difference variable with potential impact on decision making in the Centipede game is risk-taking. Previous Centipede studies manipulated the risk associated with each GO move (i.e., the amount by which the personal payoff decreased at the following node) and found that higher risk led to earlier exits in the game (e.g., Horng and Chou, 2011; Krockow, Pulford, \& Colman, 2015). Moreover, a study by Cook et al. (2005) suggests that risk-taking is likely to differ between Japanese and Western participants. Cook et al. manipulated riskiness of cooperation in Prisoner's Dilemma games by varying values of the "sucker's payoff." Japanese participants were found to cooperate less in high-risk games compared to US participants.

Finally, the current experiment was designed to assess assurance-based trust indirectly, and it does not allow for any direct conclusions regarding levels of general trust, which Yamagishi and Yamagishi (1994) defined as a spontaneously exhibited trust toward strangers in the face of social uncertainty. It is possible that general trust affects decision making in the Centipede game-particularly at the beginning of the game, which arguably yields the highest amount of social uncertainty. Since general trust was found to differ across cultures, with the Japanese scoring particularly low (Yamagishi, 1988), it is an important variable to consider in this cross-cultural study. Consequently, the present study includes measures of SVO, risktaking/risk-perception and general trust, and tests for cross-cultural differences as well as any effects on decision making in the Centipede game. Based on the studies reviewed above, we expected that SVO would not differ significantly between Japan and the UK, with results showing similar proportions of cooperative and individualistic individuals and very few individuals with extreme SVOs (altruistic and competitive). Accordingly, we formulated the following additional hypotheses:

Hypothesis 4. Japanese participants will score lower on measures of risk-taking and general trust than British participants.

Hypothesis 5. Higher levels of prosociality, risk-taking, and trust will be related to higher cooperativeness in the Centipede game.

\section{Method}


Participants. The sample comprised 100 Japanese nationals recruited at Hokkaido University Japan and 80 UK nationals recruited at the University of Leicester. All participants were undergraduate students. The Japanese sample included 63 males, 33 females, and 4 of unknown gender, with a mean age of 19.14 years $(S D=1.02)$, all of whom were remunerated with $¥ 1000$ (US\$8.14) for participation. The UK sample consisted of 15 males and 85 females, with a mean age of 19.25 years $(S D=2.71)$, who received course credits for taking part. In addition to the compensation for their time, participants in both countries were incentivized with a between-subjects random lottery incentive system (see Bolle, 1990; Cubitt, Starmer, \& Sugden, 1998). Nine participants (one per testing session) were randomly selected to receive their payoffs from a randomly chosen game completed during the experiment. The games’ payoffs were converted into the respective countries' currencies at a rate of 242.32 in Japan and 1.36 in UK to yield comparable payments. In Japan, the average lottery win per person was $¥ 5173.33$ (US\$43.72) and in the UK it was $£ 17.53$ (US\$25.33).

Design. The study used a 2 (Nationality) $\times 4$ (Treatment Condition) factorial experimental design. The participants in both countries (Japan and the United Kingdom) were randomly assigned to one of the following four treatment conditions that varied in the amount and type of information provided about the payoffs: (a) Centipede game with complete information and payoffs displayed in monetary units (Condition 1); (b) Centipede game with complete information and payoffs displayed as percentages of a pot containing a known number of monetary units (Condition 2); (c) Centipede game with absolute information about own payoffs only (Condition 3); and (d) Centipede game with relative payoff information compared to the co-player only (Condition 4), (see Figure 2). The main dependent variable was the mean exit point of the 20 games that an individual completed during the course of the experiment, with high scores indicating later exiting and therefore higher cooperativeness, and low scores indicating earlier exiting and lower cooperativeness. 


\section{(a) Condition 1: Complete information}

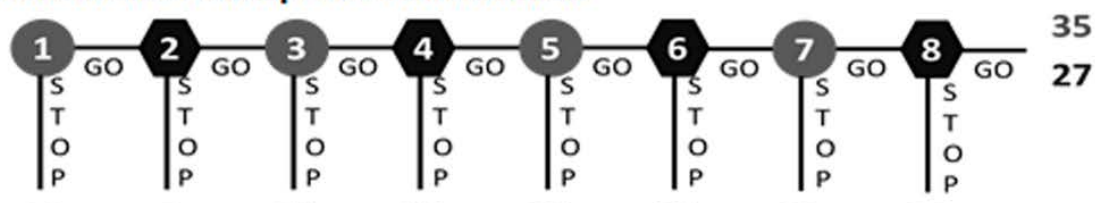

$\begin{array}{lcccccccc}\text { A } & 11 & 6 & 17 & 12 & 23 & 18 & 29 & 24 \\ \text { B } & 3 & 14 & 9 & 20 & 15 & 26 & 21 & 32\end{array}$

(b) Condition 2: Complete information (\%)

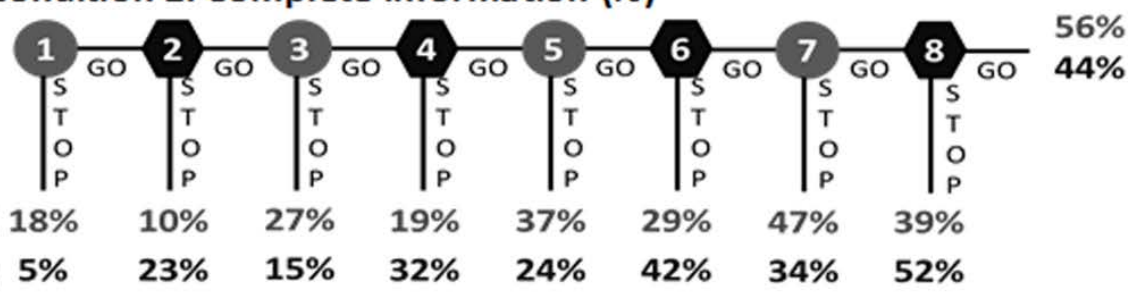

These payoffs represent percentages of a fixed pot containing 62 monetary units (worth $f 84.32$ )

(c) Condition 3a: Absolute payoff information only, perspective Player A

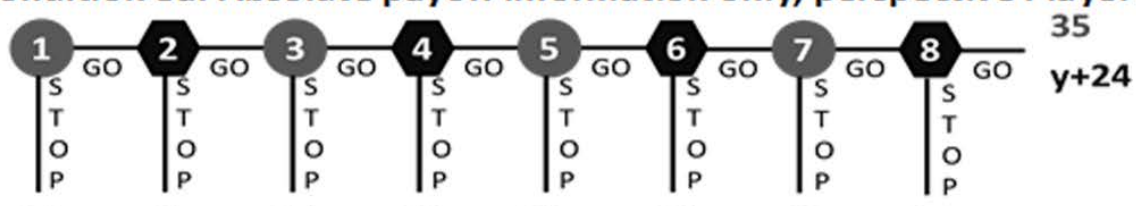
A 11
6
17
12
23
18
$29 \quad 24$
B $\begin{array}{llllllll}y & y+11 & y+6 & y+17 & y+12 & y+23 & y+18 & y+29\end{array}$

" $y$ " is fixed and remains the same throughout the experiment. It was set at a number between 0 and 10 . It is equally likely to be any of those numbers.

(c) Condition 3b: Absolute payoff information only, perspective Player B

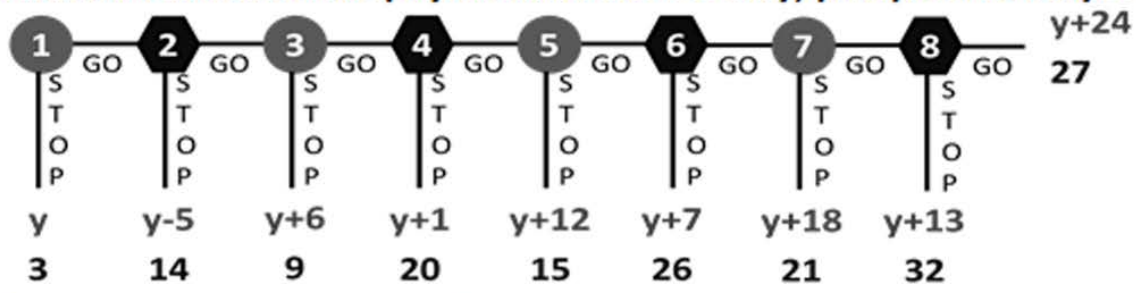

" $y$ " is fixed and remains the same throughout the experiment. It was set at a number between 4 and 15 . It is equally likely to be any of those numbers.

(d) Condition 4: Relative payoff information only

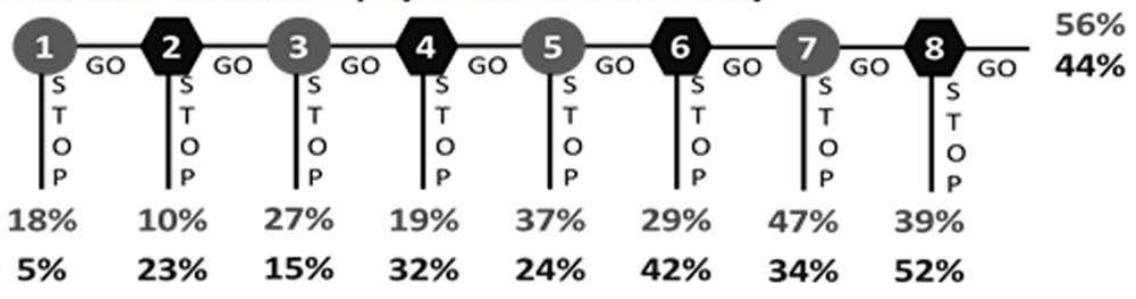

These payoffs represent percentages of a fixed pot containing an unknown number of mon. units.

Figure 2. Game trees for Conditions 1-4. 
For a behavioral measure of altruism, the number of games per individual reaching the natural end (Exit Node 9) out of 20 games in total was calculated, with higher numbers indicating higher levels of altruism. To obtain a more individual measure of cooperation, the number of exit moves made by each individual across twenty game rounds was assessed, higher numbers of exit moves suggesting lower levels of cooperation. Finally, three individual difference variables_-SVO, risk-taking/risk-perception, and trust-were computed from questionnaire measures.

Materials. The experiments were run in large computer laboratories in Japan and the UK. Participants were seated at individual computer desks - in Japan these were separated with dividing walls, in the UK by generous spacing of desks - and interacted in the Centipede game through a colorfully illustrated, custom-made web application with animated instruction pages. These pages contained the rules of the game (explained in simple and neutral lay terms) and the following information about the experiment's matching procedure:

You will be randomly paired with another participant in the room and presented with the decision sequence. The computer has randomly determined that you are Participant A [or Participant B], and you will remain as Participant A [or Participant B] throughout the experiment. You will then make decisions to GO or STOP. Once the decision sequence has ended, you will be randomly paired with a different participant and asked to make decisions to GO or STOP on the decision sequence again. The whole decision sequence will be presented 20 times in total and you will be randomly paired with a different participant for each sequence. You will not know who you are paired with.

The Japanese participants were presented with the instruction slides fully translated into Japanese. For the actual game interactions with real-time feedback, they received translation booklets containing pictures of example screens with Japanese translations of all English words and sentences. Across both countries, participants were given paper copies of the six-item SVO slider measure (Murphy, Ackermann, \& Handgraaf, 2011), the 30-item Domain-specific risktaking (DOSPERT) scale along with the related risk-perception scale (Blais \& Weber 2006), and the first five items of Yamagishi and Yamagishi's (1994) general trust scale.

The SVO score was a continuous variable with higher scores indicating increasing prosociality. In addition, the continuous SVO score was retrospectively categorized to yield traditional SVO classifications. As stipulated by the creators of the SVO slider measure (Murphy, Ackermann, \& Handgraaf, 2011), scores below -12.04 qualified as competitive, scores up to 22.45 qualified as individualistic, scores up to 57.15 qualified as cooperative, and any scores above qualified as altruistic. For risk-taking and risk-perception, five sub-scale scores each were computed for the following risk categories: ethical, financial, health/safety, recreational, and social. High scores indicate high levels of risk-taking and risk-perception respectively. The trust score was calculated by adding trust ratings across five items, with high scores representing higher trust levels.

All Japanese materials were translated from English into Japanese by our Japanese project collaborators and co-authors. The only exception was the DOSPERT scale of which a Japanese version was already available from the original authors online. Byrne (2016) raised the possible difficulty of translating psychological scales for cross-cultural research. To check the scales' reliability in both English and Japanese, we calculated the Cronbach alpha values for the three questionnaires in both countries. All scales showed high internal consistency: The alpha 
values of the SVO items measuring self-allocation were .70 in Japan and .65 in the UK. For the SVO items measuring resource allocation to the other person, the values were .70 and .71 respectively. The DOSPERT risk-taking scale produced values of .86 in Japan and .86 in the UK, and the DOSPERT risk-perception scale yielded values of 0.90 and 0.83 respectively. Finally, the Trust scale produced a Cronbach alpha of .80 in Japan and of .79 in the UK.

Procedure. The data were collected in testing sessions of exactly 20 participants at a time. Each treatment condition was run in one testing session. In Japan, an additional testing session was run for Condition 1 (standard Centipede game), because the first session contained an unusually high number of second-year students who had previously participated in other studies of experimental games and might be considered more experienced. Since no significant differences between the resulting two data sets were found, the data from these sessions were combined for the results reported in this article. Each testing session lasted between 50 and 70 minutes. The participants were told not to communicate with each other and to switch off their cell (mobile) phones. The experimenters ensured that they were focusing on their own computer screens and materials at all times.

After the participants filled in the consent form, the computer randomly assigned each participant a player role (Player A or B) in which they remained for the rest of the experiment. Participants were then presented with the computerized game instructions. The experimenters encouraged questions at all times, and answers were provided in private. After reading the instructions, the participants completed a form assessing their understanding of the game and its payoffs. The experimenters checked all replies and quietly corrected the participants in case of misunderstandings. Subsequently, all participants completed 20 rounds of Centipede games. The game repetitions were chosen to yield a larger amount of data (from 1800 individual games). After each round, the computer randomly and anonymously selected a new co-player for each participant. The web application provided real-time feedback to participants of the choices of their co-players, the game outcomes, and the number of games left to complete. After the final game round, one participant was randomly selected as winner of the lottery payment. All participants received their show-up fee or course credits at the end of the testing session. The lottery winners also received their additional payments.

\section{Results}

Comparison of exit points. A detailed overview of game terminations per exit node across treatment conditions and countries is provided in Figure 3. The game exit charts display mostly bell-shaped curves centered on varying modes depending on the treatment condition and the country. The Japanese exit curves show that Node 5 was the modal exit point in Condition 1 and Node 6 in Condition 2 (both with complete payoff information), Node 7 was modal in Condition 3 (absolute payoff information only) and, remarkably, that the final Exit Node 9 was the modal exit point in Condition 4 (relative payoff information only). In comparison with the Japanese data, the UK exit curves tend to peak more toward the left, indicating lower mean exit points and hence fewer cooperative moves. With the exception of Condition 3 (absolute payoff information only), which yielded a modal exit point of 4 , all other UK treatment conditions were most frequently terminated at Node 5. 


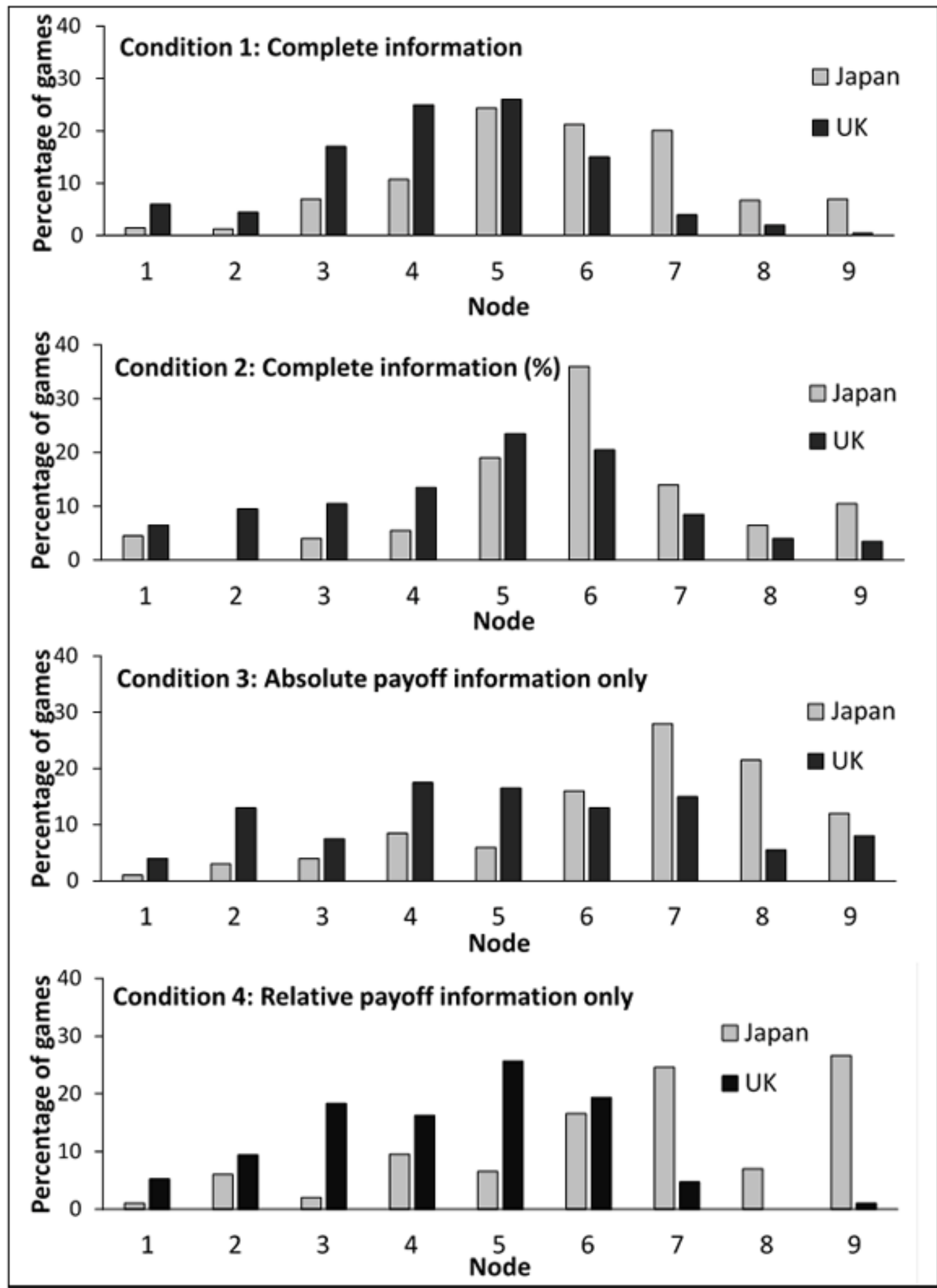

Figure 3. Percentages of games terminated at each exit point in Japan and the UK across the four treatment conditions. 
A two-way ANOVA was carried out to test for effects of nationality and treatment conditions on mean exit points in the Centipede game. Descriptive statistics for each country and treatment condition are displayed in Table 1 and Figure 4. A significant main effect was found for nationality, with the mean exit points of Japanese participants significantly higher $(M=6.13)$ than those of UK participants $(M=4.61), F(1,165)=97.452, p<.001$, partial $\eta^{2}=.46$.

Table 1

Mean Exit Points and Standard Deviations Per Country and Treatment Condition

\begin{tabular}{llllll}
\hline & $\begin{array}{l}\text { C1: Complete } \\
\text { information }\end{array}$ & $\begin{array}{l}\text { C2: Complete } \\
\text { information (\%) }\end{array}$ & $\begin{array}{l}\text { C3: Absolute } \\
\text { information } \\
\text { only }\end{array}$ & $\begin{array}{l}\text { C4: Relative } \\
\text { information } \\
\text { only }\end{array}$ & Total \\
\hline Japan & $5.78(0.91)$ & $5.94(0.99)$ & $6.55(0.79)$ & $6.61(1.37)$ & $6.13(1.01)$ \\
\hline UK & $4.35(0.63)$ & $4.75(0.82)$ & $5.04(0.85)$ & $4.31(0.60)$ & $4.61(0.78)$ \\
\hline Total & $5.28(1.07)$ & $5.34(1.08)$ & $5.80(1.11)$ & $5.40(1.46)$ & $5.44(1.18)$ \\
\hline
\end{tabular}

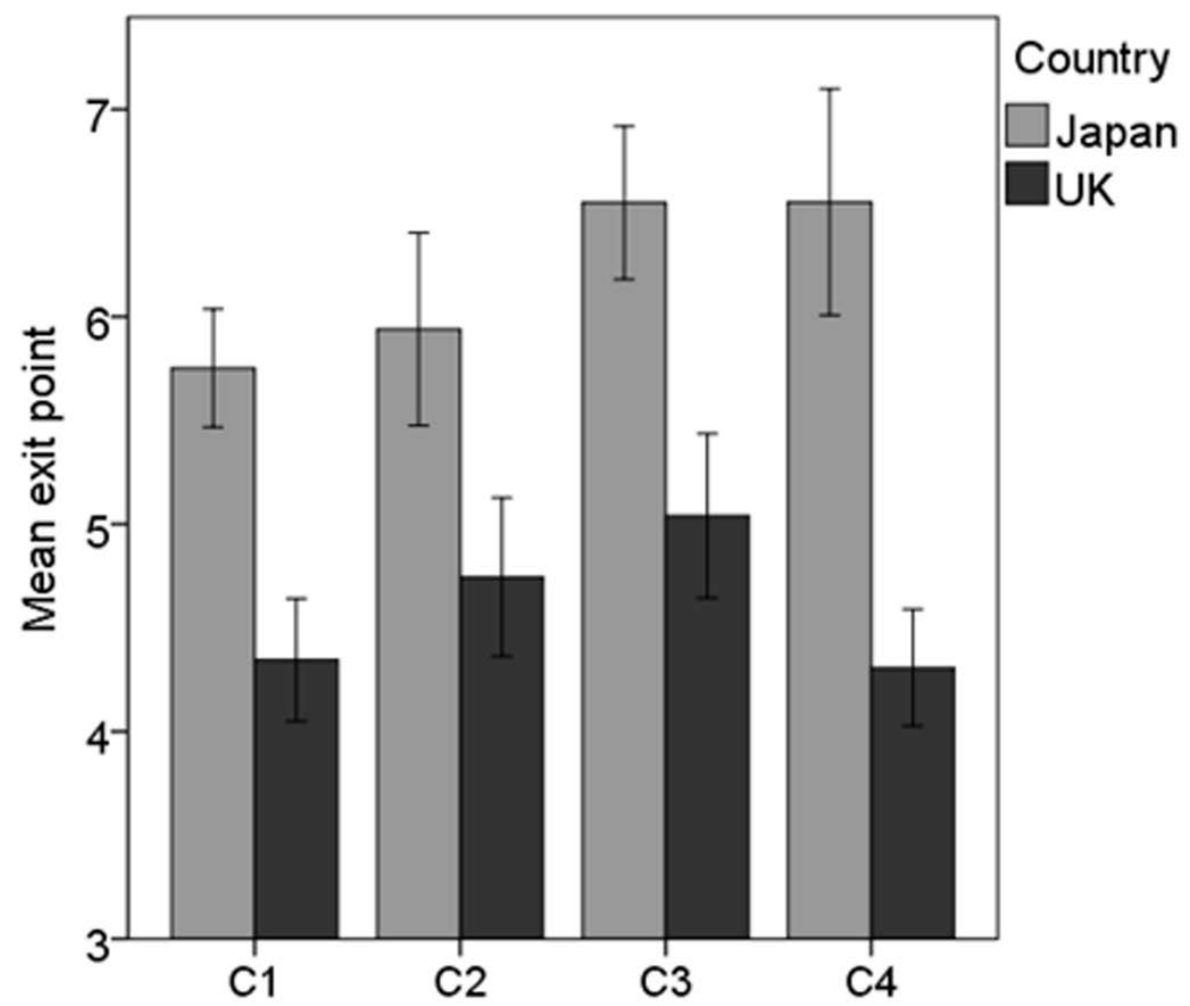

Figure 4. Mean exit points of each treatment condition in Japan and in the UK (C1: complete information; C2: complete information as percentages; C3: absolute information only; C4: relative information only). 
Treatment condition also had a significant effect: $F(3,165)=4.276, p<.005$, partial $\eta^{2}$ $=.10$. Given the additional finding of a significant interaction between nationality and treatment, $F(3,165)=2.949, p<.05$, partial $\eta^{2}=.05$, the effects of the treatment conditions varied depending on the country where the experiments were conducted. In Japan, Condition 1 (complete information) had the lowest exit points $(M=5.78)$, followed by Condition 2 (complete information in percentages) $(M=5.94)$, Condition 3 (absolute payoff information only) $(M=$ 6.55), and Condition 4 (relative payoff information only) $(M=6.61)$. Pairwise comparisons using Tukey-HSD tests indicated that the mean exit points in Condition 3 and Condition 4 were significantly higher than in Condition 1 and Condition $2(p<.05)$. Conditions 1 and 2 did not significantly differ from each other, and neither did 3 and 4 . In the UK, Condition 4 was found to have the lowest mean exit point $(M=4.31)$. This was followed by Condition $1(M=4.35)$, Condition $2(M=4.75)$, and Condition $3(M=5.04)$. Tukey-HSD post-hoc comparison showed that the mean exit point in Condition 3 was significantly higher than those in Condition 1 and Condition $4(p<.05)$. Conditions 1,2 , and 4 did not differ significantly from one another.

In order to rule out confounding gender effects, given the different gender proportions across the Japanese and the UK sample, a three-way ANOVA was conducted, testing for the effects of gender in addition to nationality and treatment condition on mean exit points. The ANOVA revealed no significant main effect of gender on mean exit points, $F(1,159)=0.011, p$ $=.918$, nor any two-way or three-way interactions including gender.

To examine factors determining altruistic behavior in the Centipede game, an additional two-way ANOVA was carried out, testing whether nationality and treatment condition affected the mean number of games per individual that reached Node 9. The means and standard deviations are displayed in Table 2 and Figure 5. A significant main effect was found for nationality, with the number of games per individual reaching Node 9 significantly higher in Japan $(M=2.52)$ than in the UK $(M=0.65), F(1,172)=33.909, p<.001$, partial $\eta^{2}=.17$.

Treatment condition was also found to have a significant effect, $F(3,172)=5.674, p$ $<.005$, partial $\eta^{2}=.09$. But again, an additional significant interaction between the two factors was found, $F(3,172)=6.896, p<.001$, partial $\eta^{2}=.107$, as evidenced by the different effects of the treatment condition depending on the nationality of the participants. In Japan, Tukey-HSD post-hoc comparison showed that Condition 4, with relative payoff information only, produced significantly more altruistic end-moves than Condition $1(p<.001)$, Condition $2(p<.01)$, and Condition 3 ( $p<.05$ ), which did not differ from one another. In the UK, it was Condition 3, with absolute payoff information, that yielded significantly more games reaching the final exit node than Condition $1(p<.001)$, Condition $2(p<.01)$, and Condition $4(p<.001)$, which did not differ significantly from one another (Tukey-HSD tests).

Table 2

Mean Number of Games Per Individual Reaching Node 9, Per Country and Treatment Condition

\begin{tabular}{llllll}
\hline & $\begin{array}{l}\text { C1: Complete } \\
\text { information }\end{array}$ & $\begin{array}{l}\text { C2: Complete } \\
\text { information } \\
\text { in \% }\end{array}$ & $\begin{array}{l}\text { C3: Absolute } \\
\text { information } \\
\text { only }\end{array}$ & $\begin{array}{l}\text { C4: Relative } \\
\text { information } \\
\text { only }\end{array}$ & Total \\
\hline Japan & $1.40(2.41)$ & $2.10(2.73)$ & $2.40(2.85)$ & $5.30(4.71)$ & $2.52(3.41)$ \\
\hline UK & $0.10(0.31)$ & $0.70(0.98)$ & $1.60(1.31)$ & $0.20(0.41)$ & $0.65(1.03)$ \\
\hline Total & $0.97(2.01)$ & $1.40(2.15)$ & $2.00(2.23)$ & $2.75(4.19)$ & $1.69(2.79)$ \\
\hline
\end{tabular}




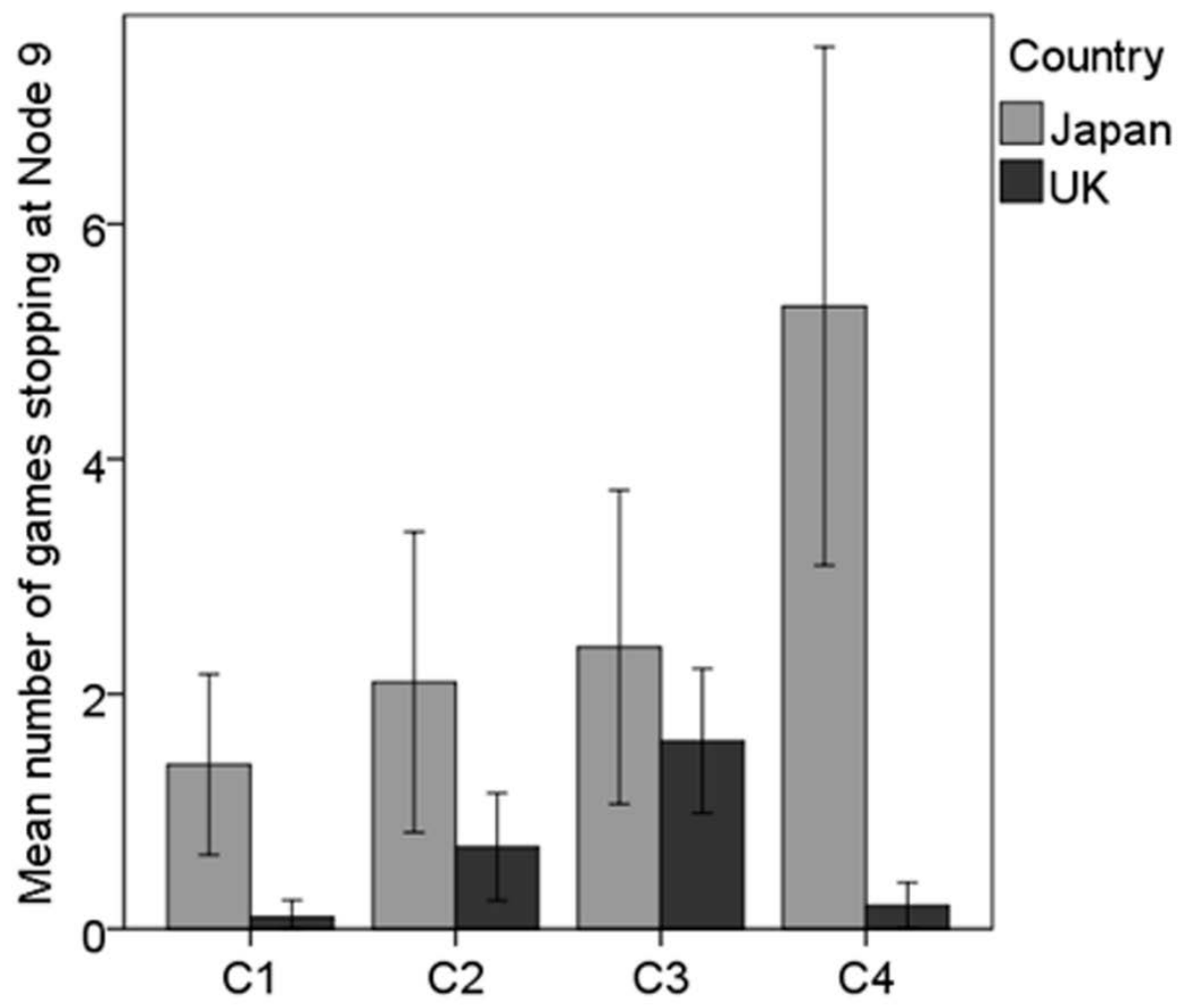

Figure 5. Mean number of games per individual reaching Node 9 across treatment conditions and country.

Analysis of individual difference variables. Several $t$ tests were conducted to compare continuous SVO scores, trust scores, and different risk-taking and risk-perception scores between Japan and the UK. For each analysis, a Levene's test for equality of variances was conducted to ensure the assumption of homogeneity of variance was met. Violations of the assumption were found only for health/safety risk-taking and recreational risk-taking, where the UK sample showed significantly larger data variances than the Japanese sample $(p<0.5)$. The $t$ test results for those variables therefore need to be interpreted with a degree of caution. The descriptive statistics are presented in Table 3. 
Table 3

Means and SD (in Parentheses) for SVO, Trust and Risk-Taking by Country

SVO, continuous

$\frac{\text { Japan Country }}{22.72(14.66) 22.54(15.5)}$

Trust

$19.43(5.55) \quad 20.7(6.37)$

\section{Risk-taking}

Ethical risk-taking

(e.g., Having an affair with a married man/woman)

Financial risk-taking

(e.g. Betting a day's income at the horse races)

$11.8(6.13) \quad 15.9(6.59)$

Health/safety risk-taking

$15.01(4.94) \quad 19.8(7.11)$

(e.g., Driving a car without wearing a seat belt)

Recreational risk-taking

$17.29(7.83) \quad 22.09(9.1)$

(e.g., Going camping in the wilderness)

$27.33(4.68) \quad 29.86(5.7)$

Social risk-taking

(e.g., Admitting that your tastes are different from those of a friend)

\section{Risk-perception}

Ethical risk-perception

$25.49(5.97) \quad 24.8(4.63)$

(e.g., Having an affair with a married man/woman)

Financial risk-perception

$30.97(7.42) \quad 30.48(6.25)$

(e.g., Betting a day’s income at the horse races)

Health/safety risk-perception

(e.g., Driving a car without wearing a seat belt)

$29.95(6.29) \quad 30.38(6.06)$

Recreational risk-perception

$26.88(6.58) \quad 26.04(6.36)$

(e.g., Going camping in the wilderness)

$18.55(5.13) \quad 17.71(5.36)$

Social risk-perception

(e.g., Admitting that your tastes are different from those of a friend)

No significant differences were found between the SVO scores, $t(178)=0.079, p=.937$, with participants of both countries producing mean scores falling within the boundaries of the cooperative SVO category but close to the individualistic category. Additionally, a chi-square 
test of independence was used to compare the numbers of participants falling within the individual SVO categories for each country. Again, no significant differences were found, $\chi^{2}(2$, $N=180)=0.936, p=.626$. In Japan, $53 \%$ of participants were categorized as cooperative, $46 \%$ as individualistic and $1 \%$ as competitive. In the UK, $56.25 \%$ of participants qualified as cooperative and the remaining $43.75 \%$ as individualistic.

Comparing the different risk-taking subscale scores, no significant differences were found between Japanese and UK participants on the ethical risk-taking subscale, $t(175)=0.033$, $p=.973$. However, on all other subscales the Japanese participants scored significantly lower than UK subjects, including financial risk-taking, $t(177)=-.304, p<.001$, health/safety risktaking, $t(176)=5.291, p<.001$, recreational risk-taking, $t(176)=-3.786, p<.001$, and social risk-taking, $t(177)=-3.259, p<.01$. As regards risk-perception on the individual sub-scales, no significant differences were found between countries. The lower risk-taking scores, in spite of similar risk-perception scores in Japanese participants, indicate higher risk-aversion of Japanese than UK participants.

With regard to the trust scores, no significant differences were found, $t(178)=-1.428, p$ $=.155$. With mean trust scores of 19.43 in Japan and 20.7 in the UK (out of a possible 35 points, indicating perfect trust), participants across both countries showed moderately high levels of general trust.

For the total sample of Japanese and UK participants, a Pearson product-moment correlation revealed a significant negative relationship between the continuous SVO score and the number of exit moves an individual made for the two treatment conditions with complete information. Both correlations were negative-Condition 1, $r=-.343, n=60, p<.01$; Condition 2, $r=-.405, n=40, p<.01$ - thus demonstrating that the number of exit moves decreased with higher (more prosocial) SVO scores. However, much weaker non-significant correlations were found for these two variables in Condition $3(r=-.183, n=40, p=.259)$ and Condition 4 ( $r=$ $-.238, n=39, p=.144)$. Also, neither trust nor any of the risk-taking scores were correlated with the number of exit moves.

\section{Discussion}

This study reports the first cross-cultural experiment on decision making in the sequential Centipede game comparing Japanese and UK nationals, and the findings relate to four treatment conditions that differed in the amount of payoff information provided. The results demonstrate significant differences in cooperation levels between the two countries and between treatment conditions. The Japanese participants exited significantly later in the game and were thus significantly more cooperative than the UK participants across all treatment conditions. With regard to the number of games reaching the final exit point - an outcome of an altruistic GO move by Player 2 at the penultimate node-the Japanese showed, once again, significantly higher scores than the UK participants. These findings support Hypothesis 1, which predicted that repeated interactions with the same co-player in the Centipede game would elicit culturally typical assurance-based trust in Japanese participants (Yamagishi \& Yamagishi, 1994), thus leading to higher levels of reciprocity, cooperation, and even altruism than among British participants.

The treatment condition (i.e., amount of payoff information provided) was also found to influence cooperation, but the effects differed between countries. In Japan, the two treatment conditions with incomplete payoff information yielded significantly later game exits and hence higher cooperation levels than those with complete information. Notably, Condition 4 (relative information only) yielded a very large percentage of games reaching the natural end (26.63\%), 
and statistical analyses revealed this result to be significantly higher than for both treatment conditions with complete information. In the UK, Condition 3, with absolute information (about own choices) only, led to a similar significant increase of cooperation compared to the standard Centipede game. It was also the condition with the highest percentage of games reaching the natural end (8\%) - significantly higher than in all other treatment conditions. In contrast with the Japanese results, however, the other incomplete information treatment condition-relative information only — did not differ significantly from the standard Centipede game and elicited, in fact, the earliest exit moves out of all four treatment conditions.

Taken together, these results corroborate Croson's (1996) findings regarding the importance of quantity and type of payoff information provided in experimental games. In line with our expectations, no significant differences between the two treatment conditions of complete information were found, demonstrating that the framing of the payoffs as either points (monetary units) or percentages did not affect decision making in the games.

However, the results strongly challenge Hypotheses 2, which predicted that incomplete information would reduce cooperation. A possible explanation could be that participants drew on previous experiences and were guided by their personal expectations about the likely stake sizes when making their choices in incomplete information games. In addition to the show-up fee, the maximum payment to be won in each session was $£ 47.60 / ¥ 8481.20$ (approximately US\$71.60)— a large amount of money for almost any university student. It is likely that both samples anticipated the stakes to be much lower. In Japan, most participants had previously participated in economic decision-making experiments with different types of games and much lower stakes (of approximately ¥1000). In the UK, most participants had never participated in experiments with real financial incentives. As with previous literature pointing to the importance of stake sizes, suggesting that lower stakes yield higher cooperation levels in the Centipede game (Rapoport et al., 2003), lower payoff expectations could explain the observed cooperativeness in games of incomplete information. This explanation is intuitively convincing for Condition 4, with relative payoff information only. In this treatment condition, the participants did not have any information about absolute payoff size which they could anchor their expectations in. If payoffs were expected to be very small, the differences between personal payoffs at individual nodes might have lost salience to participants. The more experienced Japanese participants in particular may therefore have continued their reciprocal cooperation up until the game's final end, thus explaining the high levels of behavioral altruism observed in this sample.

However, the explanation of lower payoff expectations may also- to a lesser extentapply to Condition 3, in which information on absolute payoffs of the individual was provided, thereby giving participants an idea of the personal stake size. Even though participants had accurate expectations about their own payoffs in this treatment condition, this payoff information may not have been sufficient to lower cooperation levels overall, because participants remained ignorant of their co-players' stakes. If participants expected their co-players to be incentivized with significantly lower payoffs, they may have anticipated higher cooperativeness from their co-players, in which case it would have paid off to match the level of expected cooperativeness and choose GO more frequently.

Interestingly, the UK results for Condition 4 (relative payoff information) are still unaccounted for by this explanation. It is possible that the UK participants were more sensitive to concerns of fairness and equality, which - as predicted by Hypothesis 3-may have been triggered by the relative payoff information in Condition 4. The heightened attention to player inequality could thus have counteracted the influence of lower payoff expectations, ultimately 
leading to earlier exits and lower cooperation levels than the other incomplete information treatment conditions.

As regards the individual difference measures assessed via questionnaires, as expected, no significant differences were found between the SVO scores of Japanese and British participants. The results suggest that both countries contain slightly higher percentages of cooperative than individualistic individuals. Similar to Yamagishi et al.'s (2013) findings, very few individuals with extreme orientations (altruistic or competitive) were identified in either country. Additionally, these non-significant findings support the questions raised regarding previously stated cultural differences in individualism and collectivism (e.g., Oyserman, Coon, \& Kemmelmeyer, 2002; Voronov \& Singer, 2002). If the cultural dimension of individualism (Hofstede, 2010) is indeed a meaningful concept in this context, then Japan-a nation previously identified as largely collectivist—-should surely have produced more prosocial SVO scores than the largely individualistic UK. Since this was not the case, our findings lend support to Voronov and Singer's claims that the difference between individualism and collectivism may be merely behavioral and not reflective of true differences in underlying social values.

In line with Hypothesis 4, the Japanese participants were found to be significantly more risk-averse than the UK participants on four of the five risk-taking subscales (the exception being ethical risk-taking), whereas risk-perception for these scales was comparable. However, although the Japanese scored lower than the British on the general trust questionnaire, this difference was not significant. This is in contrast with previous studies of cross-cultural differences in general trust that have consistently reported that Japanese participants produce lower scores than US participants (Yamagishi, 1988; Yamagishi \& Yamagishi, 1994). It is possible that the British culture falls in between the US and the Japanese cultures with regard to general trust but does not differ significantly from either. However, further research is necessary to corroborate this claim by conducting a more comprehensive comparison of general trust and using a larger trust scale across different cultures.

In line with Hypothesis 5 and with previous findings suggesting influences of SVO on decision making in the Centipede game (Pulford et al., 2016), continuous SVO was found to be correlated with cooperation in the game. Higher SVO scores (indicating higher prosociality) were correlated with lower numbers of exit moves (higher cooperation levels) in the two treatment conditions with complete information only. A possible reason why incomplete information games did not show the same correlation with SVO as Conditions 1 and 2 could be that the small amount of information about the other player's payoffs was insufficient to trigger prosocial concerns.

Neither risk-taking scores nor general trust were correlated with cooperativeness of the participants. The non-significant finding for risk-taking indicates that the demonstrated cultural difference in risk aversion cannot account for the observed difference in cooperation. Indeed, if risk-taking had been the decisive factor, then the risk-averse Japanese participants should have cooperated less frequently than the UK participants. General trust, the spontaneous trust exhibited when encountering a complete stranger, did not appear to play any role either. This finding lends further support to Yamagishi and Yamagishi's (1994) trust theory, according to which different types of trust may be evoked depending on the situation and the cultural background. As outlined in the Introduction, it is possible that general trust plays a negligible role in the standard Centipede game, because the players face complete strangers at the very first decision node of the game. The first move in the game (if cooperative) might therefore serve to 
establish a rapport between the players, consequently requiring assurance-based trust to maintain a reciprocal relationship.

Applying the study to real-life decision making, the findings suggests important differences in the way that Japanese and British people interpret and act in reciprocal relationships involving more than one-shot decisions, including business partnerships, that are modeled by the Centipede game. In these sequential decision contexts, the Japanese were shown to be more cooperative than the British, even in contexts with incomplete information about the possible outcomes of their actions. The Japanese appeared to form stronger reciprocal bonds from a type of assurance-based trust unique to repeated interactions with the same partner. An easily observable manifestation of this relationship approach in everyday decision making is the Asian (business) custom of reciprocal gift-giving. More research is necessary to study not only in-group decision making of different cultures but also out-group decision making. Especially with a view to further improving our understanding of international collaborations, controlled experiments on the interactions of Japanese and British participants would be fruitful. This approach could also be extended to examine cross-cultural differences in decision making between Japanese and different Western cultures. In addition to North American and UK cultures, other European cultures possibly characterized by higher levels of collectivism (e.g., Germany) could be of interest. Future studies could investigate choices across different economic games, contrasting one-shot (e.g., Prisoner's Dilemma) games necessitating general trust with sequential (e.g., Centipede) games relying on assurance-based trust.

An additional finding of this study is that incomplete information in decision-making contexts may lead to an increase of cooperation. It is possible that personal expectations, particularly regarding stake size, guide choices in decision contexts of incomplete information, with expectations of high stakes leading to lower cooperation levels, and expectations of low stakes leading to higher levels. If applied to real-life business decisions, this suggests the importance of transparency about personal aims from the outset of each interaction or partnership in order to manage confounding beliefs. In order to test for influences of different expectations directly, follow-up research could induce expectations through framed task instructions and compare subsequent decision making between treatment conditions. Alternatively, a more comprehensive account of motivations underlying decision making in the context of incomplete information could be achieved by administering qualitative self-report measures.

Limitations. Strong efforts were made to achieve comparability between the two crosscultural samples, but a few methodological limitations need to be considered when evaluating the results of this study. Due to the use of convenience samples in both countries, the gender distributions differed substantially. The Japanese sample included a much larger proportion of male participants than the UK sample. However, while this imbalance was not ideal, we found no evidence of gender effects on decision making.

Furthermore, based on local customs, compensation procedures differed between the two countries. In the Japan, all participants expected a show-up fee for attending the experiment. In the UK, a well-functioning course-credit system was in place, with most participants preferring compensation through credits rather than money. We acknowledge this difference in compensation. However, we also wish to draw attention to the fact that the crucial behavioral incentives (i.e., the money at stake during the games) was identical for both samples.

Finally, it is necessary to point out the differences in experience between the Japanese and UK students. Most Japanese students had previously participated in incentivized, economic 
experiments, whereas most UK students had not. Importantly, however, no participant in either country had previously participated in the Centipede game.

Conclusions. To conclude, this study was the first to report experimental results on cross-cultural differences in economic decision making between Japanese and British participants. The Japanese were more cooperative in repeated interactions typical of long-term business partnerships, and this could be explained by the higher levels of assurance-based trust that are characteristic of Japanese culture. Furthermore, nationals of both countries were affected by the amount of information provided about the decision context in which they operated, and they appeared to rely on previous experiences and consequent expectations when making decisions under conditions of incomplete information. Follow-up research is necessary to compare Japanese and European cultures on a wider range of decision tasks and to explore the relative importance of different types of trust.

\section{References}

Aumann, R. J. (1995). Backward induction and common knowledge of rationality. Games and Economic Behavior, 8(1), 6-19. doi:10.1016/S0899-8256(05)80015-6

Aumann, R. J. (1998). On the Centipede game. Games and Economic Behavior, 23(1), 97-105. doi:10.1006/game.1997.0605

Balliet, D., Parks, C., \& Joireman, J. (2009). Social value orientation and cooperation in social dilemmas: A meta-analysis. Group Processes Intergroup Relations, 12, 533-547. doi:10.1177/1368430209105040

Berigan, N., \& Irwin, K. (2011). Culture, cooperation, and the general welfare. Social Psychology Quarterly, 74(4), 341-360. doi:10.1177/0190272511422451

Blais, A.-R., \& Weber, E. U. (2006). A domain-specific risk-taking (DOSPERT) scale for adult populations. Judgment and Decision Making, 1, 33-47.

Bolle, F. (1990). High reward experiments without high expenditure for the experimenter. Journal of Economic Psychology,11(2), 157-167. doi:10.1016/0167-4870(90)90001-P

Byrne, B. (2016). Adaptation of assessment scales in cross-national research: Issues, guidelines, and caveats. International Perspectives in Psychology: Research, Practice, Consultation, 5(1), 51-65. doi:10.1037/ipp0000042

Christopher, M. S., \& Skillman, G. D. (2009). Exploring the link between self-construal and distress among African American and Asian American college students. Journal of College Counselling, 12(1), 44-56. doi:10.1002/j.2161-1882.2009.tb00039.x

Colman, A. M., Krockow, E. M., Frosch, C. A., \& Pulford, B. D. (2017). Rationality and backward induction in Centipede games. In N. Galbraith, E. Lucas, \& D. E. Over (Eds.), The thinking mind: A Festschrift for Ken Manktelow (pp. 139-150). London: Routledge.

Cook, K. S., Yamagishi, T., Cheshire, C., Cooper, R., Matsuda, M., \& Mashima, R. (2005). Trust building via risk taking: A cross-societal experiment. Social Psychology Quarterly, 68(2), 121-142. doi:10.1177/019027250506800202

Cox, T. H., Lobel, S. A., \& McLeod, P. L. (1991). Effects of ethnic group cultural differences on cooperative and competitive behavior on a group task. Academy of Management Journal, 34(4), 827-847. doi:10.2307/256391

Croson, R. T. A. (1996). Information in ultimatum games: An experimental study. Journal of Economic Behavior \& Organization, 30(2), 197-212. doi:10.1016/S0167-2681(96)00857-8

Cubitt, R., Starmer, C., \& Sugden, R. (1998). On the validity of the random lottery incentive system. Experimental Economics, 1(2), 115-131. doi:10.1007/bf01669298 
Fey, M., McKelvey, R. D., \& Palfrey, T. R. (1996). An experimental study of constant-sum centipede games. International Journal of Game Theory, 25, 269-287. doi:10.1007/BF02425258

Hayashi, N., Ostrom, E., Walker, J., \& Yamagishi, T. (1999). Reciprocity, trust, and the sense of control: A cross-societal study. Rationality and Society, 11, 27-46. doi:10.1177/104346399011001002

Hofstede, G. (2001a). Culture's consequences: Comparing values, behaviors, institutions, and organizations across nations (2nd Ed.). Thousand Oaks CA: Sage Publications

Hofstede, G. (2001b). Two wonderlands: Japan and the Netherlands through the looking-glass. International Scope Review, 3(5), 1-10.

Hofstede, G., Hofstede, G. J., \& Minkov, M. (2010). Cultures and organizations: Software of the mind (3rd Ed.). New York: McGraw-Hill.

Ho, D. Y. F. (1993). Relational orientation in Asian social psychology. In U. Kim \& J. W. Berry (Eds.), Indigenous psychologies: Research and experience in cultural context (pp. 240259). Newbury Park, CA: Sage.

Howard, E. S., Gardner, W. L., \& Thompson, L. (2007). The role of the self-concept and the social context in determining the behavior of power holders: self-construal in intergroup versus dyadic dispute resolution negotiations. Journal of Personality and Social Psychology, 93(4), 614-631. doi:10.1037/0022-3514.93.4.614

Kawagoe, T., \& Takizawa, H. (2008). Level-K analysis of experimental Centipede games. Journal of Economic Behavior \& Organization, 82(2), 548-566. doi:10.2139/ssrn.1289514

Kim, H., Hoskisson, R. E., \& Wan, W. P. (2004). Power dependence, diversification strategy, and performance in keiretsu member firms. Strategic Management Journal, 25(7), 613-636. doi:10.10002/smj.395

Kiyonari, T., Yamagishi, T., Cook, K. S., \& Cheshire, C. (2006). Does trust beget trustworthiness? Trust and trustworthiness in two games and two cultures: A research note. Social Psychology Quarterly, 69(3), 270-283. doi:10.1177/019027250606900304

Krockow, E. M., Pulford, B., D., Colman, A. M., (2015). Competitive Centipede games: Zeroend payoffs and payoff inequality deter reciprocal cooperation. Games, 6(3), 262-272. doi:10.3390/g6030262

Krockow, E. M., Pulford, B., D., Colman, A. M. (2016). Exploring cooperation and competition in the Centipede game through verbal protocol analysis. European Journal of Social Psychology. 46(6), 746-761. doi:10.1002/ejsp.2226

Krockow, E. M., Takezawa, M., Pulford, B. D., Colman, A. M., Smithers, S., Kita, T., \& Nakawake, Y. (2017). Commitment-enhancing tools in Centipede games: Evidencing European-Japanese differences in trust and cooperation. Unpublished manuscript, Department of Neuroscience, Psychology and Behaviour, University of Leicester, UK.

Kuwabara, K., Willer, R., Macy, M. W., Mashima, R., Terai, S., \& Yamagishi, T. (2007). Culture, identity, and structure in social exchange: A web-based trust experiment in the United States and Japan. Social Psychology Quarterly, 70(4), 461-479. doi:10.1177/019027250707000412

Mandel, N. (2003). Shifting selves and decision making: The effects of self-construal priming on consumer risk-taking. Journal of Consumer Research, 30(1), 30-40. doi:10.1086/374700

Markus, H. R., \& Kitayama, S. (1991). Culture and the self: Implications for cognition, emotion, and motivation. Psychological Review, 98(2), 224-253. doi:10.1037/0033-295X.98.2.224 
Matsumoto, D. (1999). Culture and self: An empirical assessment of Markus and Kitayama’s theory of independent and interdependent self-construals. Asian Journal of Social Psychology, 2, 289-310. doi:10.1111/1467-839x.00042

McClintock, C. G. (1972). Social motivation: A set of propositions. Behavioral Science, 17, 438-454. doi:10.1002/bs.3830170505

McKelvey, R. D., \& Palfrey, T. R. (1992). An experimental study of the Centipede game. Econometrica, 60, 803-836. doi:10.2307/2951567

Messick, D. M., \& McClintock, C. G. (1968). Motivational bases of choice in experimental games. Journal of Experimental Social Psychology, 4, 1-25. doi:10.2307/2951567

Murphy, R. O., Ackermann, K. A., \& Handgraaf, M. (2011). Measuring social value orientation. Judgment and Decision Making, 6(8), 771-781. doi:10.2139/ssrn.1804189

Murphy, R. O., Rapoport, A., \& Parco, J. E. (2006). The breakdown of cooperation in iterative real-time trust dilemmas. Experimental Economics, 9, 147-166. doi:10.1007/s10683-0067049-4.

Oyserman, D., Coon, H. M., \& Kemmelmeier, M. (2002). Rethinking individualism and collectivism: Evaluation of theoretical assumptions and meta-analyses. Psychological Bulletin, 128(1), 3-72. doi:10.1037//0033-2909.128.1.3

Pulford, B. D., Colman, A. M., Lawrence, C. L., \& Krockow, E. M. (2017). Reasons for cooperating in repeated interactions: Social value orientations, fuzzy traces, reciprocity, and activity bias. Decision. 4(2), 102-122. doi:10.1037/dec0000057

Pulford, B. D., Krockow, E. M., Colman, A. M., \& Lawrence, C. L. (2016). Social value induction and cooperation in the Centipede game. PLOS ONE, 11(3), 1-21. doi: 10.1371/journal.pone.0152352

Rapoport, A., Stein, W. E., Parco, J. E., \& Nicholas, T. E. (2003). Equilibrium play and adaptive learning in a three-person Centipede game. Games and Economic Behavior, 4, 239-265. doi:10.1016/S0899-8256(03)00009-5

Rosenthal, R. W. (1981). Games of perfect information, predatory pricing and chain store paradox. Journal of Economic Theory, 25, 92-100. doi:10.1016/0022-0531(81)90018-1

Schwartz, S. H. (1994). Beyond individualism/collectivism: New cultural dimensions of values. Los Angeles, CA: Sage.

Spencer, B. J., \& Qiu, L. D. (2001). Keiretsu and relationship-specific investment: A barrier to trade? International Economic Review, 42(4), 871-901. doi:10.1111/14682354.00138Takano, Y., \& Osaka, E. (1999). An unsupported common view: Comparing Japan and the U.S. on individualism/collectivism. Asian Journal of Social Psychology, 2, 311-341. doi:10.1111/1467-839x.00043

Tiessen, J. H. (1997). Individualism, collectivism, and entrepreneurship: A framework for international comparative research. Journal of Business Venturing, 12(5), 367-384. doi:10.1016/s0883-9026(97)81199-8

Triandis, H. C. (1995). Individualism and collectivism. Boulder, CO: Westview.

Voronov, M. \& Singer, J. A. (2002) The myth of individualism-collectivism: A critical review, Journal of Social Psychology, 142, 461-480. doi:10.1080/00224540209603912

Voyer, B. G., \& Franks, B. (2014). Toward a better understanding of self-construal theory: An agency view of the processes of self-construal. Review of General Psychology, 18(2), 101114. doi:10.1037/gpr0000003 
Wong, R. Y. M., \& Hong, Y. Y. (2005). Dynamic influences of culture on cooperation in the prisoner's dilemma. Psychological Science, 16(6), 429-434. doi:10.1111/j.09567976.2005.01552.x

Yamagishi, T. (1988a). Exit from the group and in individualistic solution to the free rider problem in the United States and Japan. Journal of Experimental Social Psychology, 24, 530-542. doi:10.1016/0022-1031(88)90051-0

Yamagishi, T. (1988b). The provision of a sanctioning system in the United States and Japan. Social Psychology Quarterly, 51, 265-271. doi:10.2307/2786924

Yamagishi, T., Mifune, N., Li, Y., Shinada, M., Hashimoto, H., Horita, Y., Miura, A., Inukai, K., Tanida, S., Kiyonari, T., Takagishi, H., Simunovic, D. (2013). Is behavioral pro-sociality game-specific? Pro-social preference and expectations of pro-sociality. Organizational Behavior and Human Decision Processes, 120, 260-271. doi:10.1016/j.obhdp.2012.06.002 Yamagishi, T., \& Yamagishi, M. (1994). Trust and commitment in the United States and Japan. Motivation and Emotion, 18, 129-166. doi:10.1007/BF02249397

\section{Footnote}

1 This prediction contradicts Croson's (1996) findings of framing effects in Ultimatum games and can be explained with reference to the inherent differences between Ultimatum and Centipede games, Ultimatum games placing an even greater emphasis on fairness and payoff equality than Centipede games. By framing payoffs as proportions of the total amount of money at stake, Responders in the Ultimatum games were reminded of their inferior player roles and their lower prospective outcomes compared to the Proposer. In the Centipede game, despite the existence of player asymmetry, the payoff inequalities are more balanced, with participants in the role of Player 1 benefitting at uneven exit nodes and participants in the role of Player 2 benefitting at even exit nodes. We therefore expected no framing effects in our games. 